\title{
Are the children of fathers whose jobs involve contact with many people at an increased risk of leukaemia?
}

\author{
Nicola T Fear, Eve Roman, Gillian Reeves, Brian Pannett
}

\begin{abstract}
Objectives-To investigate the hypothesis that children of men whose jobs involve contact with many people (particularly children) are at an increased risk of leukaemia.

Methods-A population based dataset obtained from routinely collected death certificates involving 14168 cancer deaths occurring before the age of 15 years registered in England and Wales between 1959-63 and 1970-90. Associations were assessed with the proportional cancer mortality ratio (PCMR), with all childhood cancer deaths forming the standard for comparison. The PCMRs were adjusted, by stratification, for age and year of death (in 1-year bands) and paternal social class (nine categories). Analyses were performed by estimated level of paternal occupational social contact (high, medium, and low) for all leukaemias, leukaemia subtype, age at death, year of death, and individual occupation. Results-Out of 223 occupations, 36 (16\%) were identified as having potentially high levels of social contact, and $27(12 \%)$ as having potentially medium levels of social contact. No associations were found between paternal occupational social contact and death during childhood from leukaemia (high social contact: PCMR 94, $95 \%$ confidence interval $(95 \%$ CI) 87 to 102; medium social contact: 101, 95 to 106). No associations were found when the data were analysed by leukaemia subtype, age at death, year of death, or individual occupation.

Conclusion-The findings presented here do not support the suggestion that childhood leukaemia is related to the amount of social contact that fathers experience at work.

(Occup Environ Med 1999;56:438-442)
\end{abstract}

Keywords: childhood leukaemia; paternal occupation; social contact

The suggestion that childhood leukaemia, specifically acute leukaemia, may have an infectious aetiology is not new. ${ }^{12}$ In particular, Kinlen $^{3}$ has proposed that leukaemia in children and young adults can be caused by a specific (but as yet unidentified) infection, the transmission of which is promoted by population mixing. Recently, as an extension of this idea, it has been hypothesised that offspring of men whose jobs involve contact with many people, particularly children, are at an increased risk of leukaemia. ${ }^{4}$ To date, most studies on the topic of population mixing and childhood leukaemia have either been ecological $^{35-15}$ or based on a few events. ${ }^{416}$

The findings presented here relate to over 14000 deaths from childhood cancer occurring in England and Wales between 1959-63 and 1970-90, which is the largest body of data on paternal occupation and childhood cancer ever assembled.

\section{Subjects and methods}

Routinely collected death certification data on 360640 deaths occurring before 15 years of age registered in England and Wales during 195963, 1970-78, and 1979-90 were provided for analysis in the form of anonymised individual records by the Office for National Statistics. Data were not routinely coded for 1964-9 and were, therefore, unavailable for analysis. Deaths occurring within the first 28 days of life or which had invalid information on paternal occupation, cause or year of death were excluded. Overall 167703 childhood deaths ( $47 \%$ ) were included and the present analysis is based on 14168 deaths due to cancer.

Paternal occupation, social class, and underlying cause of death were coded by the Office for National Statistics with the standard classifications in use at the time of the child's death. ${ }^{17-22}$ Paternal occupation was subsequently bridge coded to one of the 223 jobs described in the 1970 classification of occupations $^{18}$ with a bridge coding program specifically developed for use with routinely collected data. Several occupational codes were subsequently combined to allow complete bridge coding. (Further information available from BP). Cause of death was bridge coded to the 7 th revision of the international classification of diseases (ICD) ${ }^{20}$ with bridge codes developed at the London School of Hygiene and Tropical Medicine.

Sixty three $(28 \%)$ of the 223 jobs were classified as having potentially increased levels of occupational social contact by an occupational hygienist (BP): 36 being classified as high and 27 as medium (table 1). For the purposes of the present analysis, the remaining 160 (72\%), whose likely social contact was not judged to be unusual, were classified as low.

\section{STATISTICAL METHODS}

Associations between death during childhood due to leukaemia and paternal occupational social contact were assessed with the 
Table 1 Number of childhood ${ }^{\star}$ deaths from cancer and leukaemia registered among the offspring of men with potentially high or medium levels of occupational social contact, England and Wales, 1959-63, 1970-8 and 1979-90

\begin{tabular}{|c|c|c|}
\hline \multirow[b]{2}{*}{ Paternal occupation description (occupational code) (1970 revision) } & \multicolumn{2}{|c|}{ Deaths (n) } \\
\hline & Cancer & Leukaemia \\
\hline \multicolumn{3}{|l|}{ High occupational social contact: } \\
\hline Railway guards (119) & 14 & 6 \\
\hline Drivers of buses, coaches (120) & 128 & 52 \\
\hline Drivers of other road passenger vehicles (121) & 92 & 32 \\
\hline Bus conductors (131) & 29 & 12 \\
\hline Porters, ticket collectors, railway (132) & 26 & 12 \\
\hline Shop salesmen and assistants (144) & 129 & 53 \\
\hline Roundsmen (bread, milk, laundry, soft drinks) (145) & 32 & 13 \\
\hline Street vendors, hawkers (146) & 41 & 16 \\
\hline Garage proprietors (147) & 37 & 16 \\
\hline Salesmen, services, valuers, auctioneers (150) & 114 & 47 \\
\hline Police officers and men (152) & 170 & 64 \\
\hline Guards and related workers NEC (153) & 52 & 19 \\
\hline Publicans, innkeepers (154) & 42 & 13 \\
\hline Barmen (155) & 24 & 11 \\
\hline Proprietors, managers (hotels), stewards (156-158) & 17 & 7 \\
\hline Restaurateurs, waiters, counter hands (159-161) & 91 & 48 \\
\hline Hairdressers (167) & 15 & 8 \\
\hline Hospital or ward orderlies, ambulance men (170) & 24 & 5 \\
\hline Medical practitioners (qualified) (181) & 70 & 27 \\
\hline Dental practitioners (182) & 19 & 5 \\
\hline Nurses (183) & 35 & 12 \\
\hline Medical workers NEC (191) & 11 & 5 \\
\hline University teachers (192) & 31 & 17 \\
\hline Teachers $(193,194)$ & 308 & 120 \\
\hline Clergy, ministers, members of religious orders (213) & 28 & 11 \\
\hline Social welfare and related workers (215) & 32 & 12 \\
\hline Other occupations $(186-190) \dagger$ & 11 & 5 \\
\hline \multicolumn{3}{|l|}{ Medium occupational social contact: } \\
\hline Radio and radar mechanics $(24)$ & 36 & 17 \\
\hline Installers and repairmen, telephone (25) & 107 & 49 \\
\hline Electricians, electrical and electronic fitters $(27,28)$ & 285 & 115 \\
\hline Motor mechanics, auto engineers (41) & 123 & 52 \\
\hline Plumbers, fitters, heating engineers etc $(45,46)$ & 181 & 67 \\
\hline Shoemakers and shoe repairers $(61)$ & 13 & 6 \\
\hline Tailors, dress, light clothing makers (74) & 19 & 10 \\
\hline Butchers and meat cutters (79) & 72 & 28 \\
\hline Painters, decorators NEC (100) & 209 & 77 \\
\hline Postmen, mail sorters (129) & 112 & 45 \\
\hline Messengers (130) & 12 & 7 \\
\hline Clerks, cashiers, office machine operators $(139,140)$ & 637 & 286 \\
\hline Proprietors and managers, sales (143) & 447 & 189 \\
\hline Commercial travellers, manufacturers' agents (148) & 232 & 112 \\
\hline Fire brigade officers and men (151) & 57 & 22 \\
\hline Valets and related service workers $(164)$ & 21 & 9 \\
\hline Office cleaners, window cleaners (166) & 43 & 12 \\
\hline Launderers, dry cleaners, and pressers (168) & 13 & 6 \\
\hline Proprietors and managers, service and recreation (171) & 14 & 8 \\
\hline Service, sport, and recreation workers NEC (172) & 47 & 21 \\
\hline Stage managers, actors, entertainers, etc (207) & 25 & 12 \\
\hline Judges, barristers, advocates, solicitors (214) & 47 & 25 \\
\hline Other occupations $(49,185) \dagger$ & 11 & 5 \\
\hline Total & 4385 & 1828 \\
\hline
\end{tabular}

${ }^{\star}$ Excludes deaths occurring within 28 days of birth.

$\uparrow$ Those occupations based on $<10$ cancer deaths.

$\mathrm{NEC}=$ not elsewhere classified. proportional cancer mortality ratio (PCMR), with all childhood cancer deaths forming the standard for comparison. PCMRs were adjusted, by stratification, for age and year of death (in 1-year bands) and paternal social class (nine categories). For each PCMR, approximate $95 \%$ confidence intervals (95\% CIs) and two sided tests of significance were estimated from the $\chi^{2}$ distribution, or when the number of observed deaths was $<10$, from the Poisson distribution. ${ }^{23}$

\section{Results}

The PCMRs for leukaemia by estimated level of paternal occupational social contact are shown in table 2 . Overall, for leukaemia, the PCMRs were 94 (95\% CI 87 to 102, based on 648 deaths) for high social contact, 101 (95\% CI 95 to 106, based on 1180 deaths) for medium social contact and 101 (95\% CI 98 to 104 , based on 4062 deaths) for low social contact. Data were further examined by age (0-4 years and 5-14 years) and period (1959-63, 1970-8, and 1979-90). There was no evidence of a difference between the estimates of risk by either age or period for each level of social contact. Table 2 also shows that no notable patterns emerged when these data were analysed by leukaemia subtype.

The figure shows the job specific PCMRs for leukaemia for children whose fathers' occupations are listed in table 1 . The job specific PCMRs are plotted as black squares, each with an area proportional to the square root of the number of deaths on which it is based with a central horizontal line corresponding to the 95\% CI. The overall PCMR for each level of exposure is depicted as a diamond, its height being proportional to the square root of the number of deaths on which it is based and its width corresponding to the $95 \%$ CI. Occupations with $<10$ deaths from leukaemia have not been presented graphically but have been included in the overall estimates. In the high social contact group, two occupations (chiropodists and physiotherapists (occupational codes 187 and 188)) had 0 deaths observed and expected from leukaemia, and therefore did not contribute any information to the overall estimate of risk.

Table 2 Adjusted proportional cancer mortality ratios (PCMRs)* $(95 \%$ CIs) for childhood leukaemia deathst registered among the offspring of men with social contact at work $\neq$, England and Wales, 1959-63, 1970-8, and 1979-90

\begin{tabular}{|c|c|c|c|c|c|c|}
\hline & \multicolumn{2}{|c|}{ High social contact } & \multicolumn{2}{|c|}{ Medium social contact } & \multicolumn{2}{|c|}{ Low social contact } \\
\hline & Deaths & $P C M R^{\star}(95 \% C I)$ & Deaths & $P C M R^{\star}(95 \% C I)$ & Deaths & $P C M R^{\star}(95 \% C I)$ \\
\hline All leukaemias (ICD-7:204) & 648 & $94(87$ to 102$)$ & 1180 & $101(95$ to 106$)$ & 4062 & 101 (98 to 104$)$ \\
\hline \multicolumn{7}{|l|}{ Ages (y): } \\
\hline $0-4$ & 212 & 95 (83 to 108$)$ & 426 & $103(93$ to 113$)$ & 1433 & $100(95$ to 105$)$ \\
\hline $5-14$ & 436 & $94(86$ to 104$)$ & 754 & $99(93$ to 107$)$ & 2629 & $101(97$ to 105$)$ \\
\hline \multicolumn{7}{|l|}{ Period: } \\
\hline $1959-63$ & 173 & $101(87$ to 117$)$ & 358 & 98 (89 to 109$)$ & 1199 & $100(95$ to 106$)$ \\
\hline 1970-78 & 261 & $90(80$ to 102$)$ & 496 & $102(94$ to 112$)$ & 1754 & $101(96$ to 106$)$ \\
\hline $1979-90$ & 214 & 95 (83 to 108$)$ & 326 & $100(90$ to 112$)$ & 1109 & $101(95$ to 107$)$ \\
\hline \multicolumn{7}{|l|}{ Subtype: } \\
\hline Lymphatic leukaemia (ICD-7: 204.0) & 321 & $92(83$ to 103$)$ & 565 & $101(93$ to 109$)$ & 1954 & $101(97$ to 106$)$ \\
\hline Myeloid leukaemia (ICD-7: 204.1) & 137 & $100(84$ to 118$)$ & 209 & $104(91$ to 119$)$ & 716 & $99(92$ to 106$)$ \\
\hline Monocytic leukaemia (ICD-7:204.2) & 11 & 75 (42 to 136$)$ & 31 & $109(76$ to 154$)$ & 89 & $101(82$ to 125$)$ \\
\hline $\begin{array}{l}\text { Other and unspecified leukaemia (ICD-7: } \\
204.3-204.9 \text { ) }\end{array}$ & 179 & $96(83$ to 112$)$ & 375 & 98 (88 to 108$)$ & 1303 & 101 (96 to 107$)$ \\
\hline
\end{tabular}

^PCMRs are adjusted for age at death, year of death, and paternal social class. Using all childhood cancer deaths as the standard for comparison. †Excludes deaths occurring within 28 days of birth.

‡Occupations included within the high and medium social contact groups are shown in table 1, the remaining codes comprise the low social contact group. 


\begin{tabular}{|c|c|c|c|c|}
\hline $\begin{array}{l}\text { Paternal occupation } \\
\text { (1970 revision) }\end{array}$ & Deaths & PCMR & $\begin{array}{c}\text { PCMR } \\
(95 \% \mathrm{Cl})\end{array}$ & \\
\hline \multicolumn{5}{|l|}{ High occupational exposure: } \\
\hline Drivers of buses, coaches (120) & 52 & 93 & & \\
\hline Drivers of other road passenger vehicles (121) & 32 & 89 & & \\
\hline Bus conductors (131) & 12 & 94 & & \\
\hline Porters, ticket collectors, railway (132) & 12 & 124 & & \\
\hline Shop salesmen and assistants (144) & 53 & 90 & & \\
\hline Roundsmen (bread, milk, laundry, soft drinks) (145) & 13 & 105 & & \\
\hline Street vendors, hawkers (146) & 16 & 94 & & \\
\hline Garage proprietors (147) & 16 & 99 & & \\
\hline Salesmen, services, valuers, auctioneers (150) & 47 & 94 & & \\
\hline Police officers and men (152) & 64 & 84 & & \\
\hline Guards and related workers NEC (153) & 19 & 97 & & \\
\hline Publicans, innkeepers (154) & 13 & 88 & & \\
\hline Barmen (155) & 11 & 105 & & \\
\hline Restauranteurs, waiters, counter hands (159-161) & 48 & 117 & & \\
\hline Medical practitioners (qualified) (181) & 27 & 90 & & \\
\hline Nurses (183) & 12 & 91 & & \\
\hline University teachers (192) & 17 & 110 & & \\
\hline Teachers $(193,194)$ & 120 & 94 & & \\
\hline Clergy, ministers, members of religious orders (213) & 11 & 101 & & \\
\hline Social welfare and related workers (215) & 12 & 92 & & \\
\hline All high & 648 & 94 & & \\
\hline \multicolumn{5}{|l|}{ Medium occupational exposure: } \\
\hline Radio and radar mechanics (24) & 17 & 108 & & \\
\hline Installers and repairmen, telephone (25) & 49 & 110 & & \\
\hline Electricians, electrical and electronic fitters $(27,28)$ & 115 & 99 & & \\
\hline Motor mechanics, auto engineers (41) & 52 & 104 & & \\
\hline Plumbers, fitters, heating engineers, etc $(45,46)$ & 67 & 88 & & \\
\hline Tailors, dress, light clothing makers (74) & 10 & 134 & & \\
\hline Butchers and meat cutters (79) & 28 & 89 & & \\
\hline Painters, decorators NEC (100) & 77 & 87 & & \\
\hline Postmen, mail sorters (129) & 45 & 103 & & \\
\hline Clerks, cashiers, office machine operators $(139,140)$ & 286 & 101 & & \\
\hline Proprietors and managers, sales (143) & 189 & 102 & & \\
\hline Commercial travellers, manufacturers' agents (148) & 112 & 111 & & \\
\hline Fire brigade officers and men (151) & 22 & 91 & & \\
\hline Office cleaners, window cleaners (166) & 12 & 81 & & \\
\hline Service sport and recreation workers NEC (172) & 21 & 101 & & \\
\hline Stage managers, actors, entertainers, etc (207) & 12 & 108 & & \\
\hline Judges, barristers, advocates, solicitors (214) & 25 & 104 & & \\
\hline \multirow[t]{2}{*}{ All medium } & 1180 & 101 & & \\
\hline & & ${ }_{50}^{\llcorner}$ & $\frac{Y}{100}$ & $\frac{1}{200}$ \\
\hline
\end{tabular}

PCMR = proportional cancer mortality ratio; NEC = not elsewhere classified.

Number of deaths, adjusted proportional cancer mortality ratios (PCMRs) and 95\% confidence intervals (95\% CI) for deaths from childhood leukaemia by paternal occupation for those paternal occupations with potentially high or medium levels of social contact. Occupations with $<10$ deaths from leukaemia have not been presented graphically but have been included in the overall estimates.

Of particular interest are those occupations involving frequent contact with children and young adults - for example, university teachers (occupational code 192) and school teachers (occupational codes 193 and 194) -in which the PCMRs for leukaemia among the offspring of these men were 110 (95\% CI 68 to 177 , based on 17 deaths) and 94 (95\% CI 79 to 113 , based on 120 deaths) respectively.

\section{Discussion}

The findings presented here do not support the suggestion that leukaemia in children is related to the amount of social contact that fathers 
Table 3 Adjusted proportional cancer mortality ratios (PCMRs)* (95\% CIs) for childhood leukaemia deathst registered among the offspring of men with social contact at work (Kinlen's classification ${ }^{4}$ ), England and Wales, 1959-63, 1970-8, and 1979-90

\begin{tabular}{|c|c|c|c|c|c|c|}
\hline & \multicolumn{2}{|c|}{ Very high social contact } & \multicolumn{2}{|c|}{ Medium social contact } & \multicolumn{2}{|c|}{ Low social contact } \\
\hline & Deaths & $P C M R^{\star}(95 \% C I)$ & Deaths & $P C M R^{\star}(95 \% C I)$ & Deaths & $P C M R^{\star}(95 \% C I)$ \\
\hline Leukaemia (204) & 137 & $96(81$ to 113$)$ & 2298 & 99 (95 to 104$)$ & 3455 & $101(97$ to 104$)$ \\
\hline Lymphatic leukaemia (204.0) & 75 & $91(72$ to 114$)$ & 11112 & $98(93$ to 104$)$ & 1653 & $102(97$ to 107$)$ \\
\hline Myeloid leukaemia (204.1) & 32 & $122(86$ to 172$)$ & 438 & $102(93$ to 112$)$ & 592 & $97(90$ to 106$)$ \\
\hline Monocytic leukaemia (204.2) & 0 & - & 46 & $91(68$ to 121$)$ & 85 & $111(89$ to 137$)$ \\
\hline Other and unspecified leukaemia (204.3-204.9) & 30 & 97 (68 to 139$)$ & 702 & $100(93$ to 107$)$ & 1125 & $100(95$ to 106$)$ \\
\hline
\end{tabular}

^PCMRs are adjusted for age at death, year of death, and paternal social class, with all childhood cancer deaths as the standard for comparison.

tExcludes deaths occurring within 28 days of birth.

experience at work. Of note is the lack of an association for occupations involving frequent mixing with children and young adults - such as school and university teachers-as it has been suggested that the children of such men would have the highest risk. ${ }^{4}$

The findings described in this paper have the advantage of being based on individual records obtained from a very large population based dataset derived from routinely collected childhood death certification data. Furthermore, cause of death and paternal occupation would have been recorded without bias, as the registrar and parents would have been unaware of the use of these data at the time of registering the death. Also, the classification of occupations by their potential level of social contact was constructed before the start of the study in collaboration with an occupational hygienist (BP).

However, as this study is based on cancer deaths, as opposed to incident cancers, it is important to consider the influence of changes in survival. Over the past few decades survival rates for all types of childhood cancer have improved particularly for acute lymphocytic leukaemia, for which the 5 year survival rate has risen from $2 \%(1954-63)$ to $73 \%$ $(1986-88) .^{2425}$ Under such circumstances, mortality data are not ideal for determining risk factors of childhood cancer. As the estimates of risk were similar for each period considered it seems unlikely that changes in survival have influenced the results.

In all proportional analyses, the estimates of risk may be unduly influenced by the most common causes of death. ${ }^{26}$ The PCMR removes the influence of these causes as the standard for comparison is restricted to cancer deaths only. In these data the other causes of death were shown to unduly influence the estimates of risk for this exposure group (data not shown), therefore making it inappropriate to use proportional mortality ratios (where the standard for comparison is based on all causes of death).

The use of occupational title as a proxy measure of potential exposure is controversial but is the only approach available for use with these data. The two previously conducted studies $^{416}$ used a similar approach. It is important to note that father's occupation at the time of the child's death rather than that held before conception, during pregnancy, or at the time of birth has been examined in this study. Also, the influence of variables such as child's sex and ethnicity could not be examined as this information was not provided by the Office for National Statistics.

To our knowledge only two, much smaller, studies have published data on childhood leukaemia and parental occupational social contact. ${ }^{416}$ Both examined data from geographical areas with either higher than average rates of childhood leukaemia or unusual patterns of population mixing. One found no evidence of an association with either paternal or maternal occupational social contact $^{16}$ whereas the other reported a significant positive trend $(p<0.001)$ with increasing levels of paternal occupational social contact (adjusted observed to expected ratios of 1.00, 1.75 , and 2.17 for medium and low, high, and very high paternal occupational social contact, respectively). ${ }^{4}$ This study was based on the re-analysis of five previously published studies. ${ }^{5-9} 11$ It was unclear what factors were taken into account in the adjustment procedures and it is possible that Kinlen's findings reflect residual confounding by social class or some other factor.

No significant associations were found when data were analysed for the whole of England and Wales for a 26 year period (as shown here) or when a small subset of these data (1970-2) were analysed by rural or urban status. ${ }^{4}$ Exclusion of deaths occurring in 1970-2 from the analyses presented here had no effect on the results (data not shown). The data presented here could not be used to examine geographical patterns but it may be possible to do so with data from the United Kingdom childhood cancer study (UKCCS). This is a collaborative case-control study based on several thousand incident cases of childhood cancer diagnosed before 15 years of age within the United Kingdom.

The occupational groupings used in the studies by Roman et $a l^{16}$ and Kinlen ${ }^{4}$ differed from each other and from the one presented here; this difference being partly due to the occupational coding schemes used. The application of these schemes to our data had no effect on the observed results. This is particularly important for Kinlen's study ${ }^{4}$ (table 3). The main difference between Kinlen's classification (which was based on the 1960 classification of occupations ${ }^{17}$ ) and the one used here, relates to the inclusion of additional occupations in the high social contact group - such as certain administrators and managers, construction workers, the armed forces, and certain transport workers. Kinlen also created a very high social contact group that consisted of occupations involving frequent contact with 
children or young adults. The analyses presented in this paper had already been completed for a doctoral thesis (NTF) before the publication of Kinlen's paper. ${ }^{4}$

Kinlen's hypothesis ${ }^{4}$ that the offspring of men whose jobs involve contact with many people are at an increased risk of leukaemia assumes that these men expose their children to a specific leukaemogenic agent acquired within the working environment. Although infectious agents may indeed be involved in leukaemogenesis, ${ }^{12}$ the lack of an association in these analyses is reassuring and leads us to think that there is no relation between childhood leukaemia and the amount of social contact that fathers experience at work.

We thank the Office for National Statistics for supplying the childhood death certification data for epidemiological analysis, Leslie Styles from the Medical Research Council Environmental Epidemiology Unit in Southampton for the development of the occupational recoding program, Paul Appleby for producing the figure and Krys Baker for help with the occupational recoding, both from the Imperial Cancer Research Fund, Cancer Epidemiology Unit in Oxford. NTF carried out this work as part of her doctoral thesis while based at the Imperial Cancer Research Fund, Cancer Epidemiology Unit in Oxford.

1 Greaves MF. Aetiology of acute leukaemia. Lancet 1997;349:344-9.

2 Kinlen LJ. Epidemiological evidence for an infective basis in childhood leukaemia. Br f Cancer 1995;71:1-5.

3 Kinlen LJ. Evidence for an infective cause of childhood leukaemia: comparison of a Scottish new town with nuclear reprocessing sites in Britain. Lancet 1988;2:1323-7.

4 Kinlen LJ. High-contact paternal occupations, infection and childhood leukaemia: five studies of unusual populationchildhood leukaemia: five studies of unusual popt
mixing of adults. Br $\mathcal{F}$ Cancer 1997;76:1539-45.

mixing of adults. Br f Cancer 1997;76:1539-45.
5 Kinlen LJ, Clarke K, Hudson C. Evidence from population mixing in British new towns 1946-85 of an infective basis mixing in British new towns 1946-85 of an infective
for childhood leukaemia. Lancet 1990;336:577-82.

6 Langford I. Childhood leukaemia mortality and population changes in England and Wales 1969-73. Soc Sci Med 1991; 33:435-40.

7 Kinlen LJ, Hudson C. Childhood leukaemia and poliomyelitis in relation to military encampments in England and Wales in the period of national military service, 1950-63. BMF 1991;303:1357-62.

8 Kinlen LJ, Hudson CM, Stiller CA. Contacts between adults as evidence for an infective origin of childhood leukaemia: an explanation for the excess near nuclear establishments in west Berkshire? Br $\mathcal{F}$ Cancer 1991;64 549-54.
9 Kinlen LJ, O'Brien F, Clarke K, et al. Rural population mixing and childhood leukaemia: effects of the North Sea oil industry in Scotland, including the area near Dounreay nuclear site. BMF 1993;306:743-8.

10 Kinlen LJ, John SM. Wartime evacuation and mortality from childhood leukaemia in England and Wales in 19459. BMF 1994;309:1197-202.

11 Kinlen LJ, Dickson M, Stiller CA. Childhood leukaemia and non-Hodgkin's lymphoma near large rural construction sites, with a comparison with Sellafield nuclear site. tion sites, with a compar

12 Kinlen LJ, Petridou E. Childhood leukemia and rural population movements: Greece, Italy, and other countries. Cancer Causes Control 1995;6:445-50.

13 Stiller CA, Boyle PJ. Effect of population mixing and socioeconomic status in England and Wales, 1979-85, on lymphoblastic leukaemia in children. BMf 1996;313:1297300 .

14 Dockerty JD, Cox B, Borman B, et al. Population mixing and the incidence of childhood leukaemias: retrospective
comparison in rural areas of New Zealand. BMF 1996;312: comparison.

15 Alexander FE, Chan LC, Lam TH, et al. Clustering of childhood leukaemia in Hong Kong: association with the childhood peak and common acute lymphoblastic leukaemia and with population mixing. Br f Cancer 1997;75:45763.

16 Roman E, Watson A, Bull D, et al. Leukaemia risk and social contact in children aged 0-4 years in southern England. $\mathcal{F}$ Epidemiol Community Health 1994;48:601-2.

17 Registrar General. Classification of occupations 1960 London: The Stationery Office, 1960

18 Office of Population Censuses and Surveys. Classification of occupations 1970. London: The Stationery Office, 1970

19 Office of Population Censuses and Surveys. Classification of Occupations 1980. London: The Stationery Office, 1980

20 World Health Organisation. International classification of diseases: 7 th ed. Manual of the international statistical classification of diseases

21 World Health Organisation. International classification of diseases: 8th ed. Manual of the international statistical classification of diseases, injuries and causes of death. Vol 1. Geneva: cation of diseases,

22 World Health Organisation. International classification of diseases: 9th edition. Manual of the international statistical classification of diseases, injuries and causes of death. Vol 1. Geneva: WHO, 1977.

23 Breslow NE, Day NE. Statistical methods in cancer research. Volume II - the design and analysis of cohort studies. Lyon: International Agency for Research on Cancer, 1987. (Sci publ no 82.)

24 Birch JM, Marsden HB, Jones PH, et al. Improvements in survival from childhood cancer: results of a population survival from childhood cancer: results of a pop
based survey over 30 years. $B M \mathcal{F} 1988 ; 296: 1372-6$.

25 Stiller CA. Population based survival rates for childhood cancer in Britain, 1980-91. BMF 1994;309:1612-16.

26 McDowall M. Adjusting proportional mortality ratios for the influence of extraneous causes of death. Stat Med 1983; 2:467-475. 